\title{
Las unidades de hemodiálisis en México: una evaluación de sus características, procesos y resultados
}

\author{
Laura Leticia Tirado-Gómez, MC, M en C, D en C, ${ }^{(1,2)}$ Juan Luis Durán-Arenas, MC, PhD, (I) \\ Mario Enrique Rojas-Russell, Lic en Psicol, M en C, D en C, ${ }^{(1,3)}$ Aída Venado-Estrada, MC,(I) \\ Reyna Lizzete Pacheco-Domínguez, MC, M en C, (I) Malaquías López-Cervantes, MC, PhD.(I)
}

\begin{abstract}
Tirado-Gómez LL, Durán-Arenas JL, Rojas-Russell ME, Venado-Estrada A, Pacheco-Domínguez RL, López-Cervantes M. Las unidades de hemodiálisis en México: una evaluación de sus características, procesos y resultados. Salud Publica Mex 20II;53 supl 4:S49I-S498.
\end{abstract}

\section{Resumen}

Objetivo. Evaluar las características, procesos y resultados, así como, la estructura de una muestra de unidades de hemodiálisis (UH) en México. Material y métodos. Estudio transversal en $83 \mathrm{UH}$ públicas y privadas del país. Las UH fueron estratificadas, de acuerdo con el puntaje obtenido, en cinco categorías: muy buena, buena, regular, mala y muy mala. Resultados. El $48 \%$ de las UH se clasificaron en las categorías mala y muy mala y atendieron $58 \%$ de los pacientes. El promedio de sesiones/paciente en los seis meses evaluados fue de I. 2 por semana. El $46.5 \%$ de las sesiones con determinación de $\mathrm{Kt} / \mathrm{V}$ reportaron valores inferiores al valor recomendado $(\mathrm{Kt} / \mathrm{V} \geq \mathrm{I}$.2). En infraestructura, tres cuartas partes de las unidades obtuvieron puntajes menores a $70 \%$. Conclusiones. La mitad de las unidades de hemodiálisis no cumplieron los criterios de proceso y resultado establecidos por el Consejo de Salubridad de la Secretaría de Salud.

Palabras clave: hemodiálisis; infraestructura; evaluación de procesos y resultados; México
Tirado-Gómez LL, Durán-Arenas JL, Rojas-Russell ME,

Venado-Estrada A, Pacheco-Domínguez RL, López-Cervantes M. An evaluation of the characteristics, processes and outcomes of hemodialysis units in Mexico. Salud Publica Mex 20I I;53 suppl 4:S49I-S498.

\section{Abstract}

Objective. To evaluate the characteristics, processes, outcomes and structure of a sample of hemodialysis units $(\mathrm{HU})$ in Mexico. Material and Methods. Cross-sectional study in 83 public and private $\mathrm{HU}$ from the 32 states in Mexico. The HU were stratified, according to a score, in five categories: very good, good, regular, poor and very poor. Results. $48 \%$ of the $\mathrm{HU}$ were classified as poor and very poor, and they granted medical attention to $58 \%$ of the patients. The average number of sessions per patient in the last six months assessed was I. 2 per week. $46.5 \%$ of the sessions with determination of $\mathrm{Kt} / \mathrm{V}$ reported values inferior to the recommended value $(\mathrm{Kt} /$ $V \geq 1.2$ ). $75 \%$ of the units scored less than $70 \%$ in infrastructure. Conclusions. Half of the HU did not fulfill the process and result criteria defined by the Consejo de Salubridad de la Secretaría de Salud.

Key words: hemodialysis; infrastructure; outcome and process assessment; Mexico

(I) Facultad de Medicina, Universidad Nacional Autónoma de México. México.

(2) Instituto Nacional de Pediatría. México.

(3) Facultad de Estudios Superiores Zaragoza, Universidad Nacional Autónoma de México. México.

Fecha de recibido: 30 de agosto de 2010 - Fecha de aceptado: 7 de septiembre de 201I

Autor de correspondencia: Dr. Malaquías López Cervantes. Unidad de Proyectos Especiales de Investigación, Facultad de Medicina. Torre de investigación, Ier. piso, Ciudad Universitaria. 04510 Delegación Coyoacán, México, DF, México.

Correo electrónico: mlopez@liceaga.facmed.unam.mx; mlopezl4@unam.mx 
L hemodiálisis es un tratamiento relativamente nuevo en México pues se desarrolló durante la última década del siglo XX. Anteriormente, todos los pacientes con enfermedad renal crónica recibían tratamiento de sustitución a través de la diálisis peritoneal. Este procedimiento permite remover las toxinas y el exceso de líquidos del cuerpo mediante el uso de una máquina y un dializador, también conocido como riñón artificial.

Actualmente, México no cuenta con un registro nacional de enfermos renales crónicos, pero si aplicamos el porcentaje promedio de habitantes enfermos en otros países (que equivale al $0.1 \%$ de la población), podemos estimar que hay más de 102000 enfermos renales crónicos en el país, ${ }^{1}$ de los cuales sólo 37000 cuentan con algún tratamiento sustitutivo de manera continua. El $80 \%$ de estos enfermos son atendidos por el IMSS o el ISSSTE, sin embargo, este porcentaje tiene un crecimiento anual de $11 \%$, lo que representa una demanda de servicios de hemodiálisis claramente elevada. ${ }^{2}$ Además, la hemodiálisis sigue siendo poco accesible; de cada 10 pacientes, ocho reciben diálisis peritoneal y únicamente dos reciben hemodiálisis. ${ }^{3}$

En México no existen evaluaciones sobre el funcionamiento y la estructura de las unidades de hemodiálisis. Por ello, el presente trabajo tiene como objetivo evaluar las características, procesos y resultados, así como la estructura de las unidades de hemodiálisis, utilizando tanto criterios nacionales como internacionales, apegados a la normatividad o lineamientos existentes y aplicables a este tipo de establecimientos. Esto con el propósito de generar un diagnóstico de funcionamiento de las unidades de hemodiálisis que permita proponer las guías de evaluación de dichas unidades a nivel nacional.

\section{Material y métodos}

Se realizó un estudio transversal de las unidades médicas con servicio de hemodiálisis en las 32 entidades federativas del país con el fin de identificar sus aspectos estructurales, logísticos y organizacionales, así como sus resultados.

Se identificaron 312 unidades de hemodiálisis y a partir de ellas se tomó una muestra por conveniencia de 27\% (83 unidades). Para recolectar información se emplearon cuatro cédulas elaboradas con base en los criterios de procesos y resultados para evaluación de establecimientos de atención médica de hemodiálisis del Consejo de Salubridad General ${ }^{4}$ y en las Guías de los centros de hemodiálisis elaboradas por la Sociedad Médica Española; ${ }^{5-7}$ las primeras dos cédulas permitieron recolectar información de tipo primario, y las últimas dos información de tipo secundario.
Con la primera cédula se evaluaron las características de estructura de cada unidad. Incluyó 272 preguntas, agrupadas en 23 indicadores. La segunda cédula valoró los procesos y resultados de las unidades de hemodiálisis a través de 193 preguntas distribuidas en 19 indicadores. La tercera, con 132 preguntas, evaluó los registros de información secundaria existentes sobre los pacientes atendidos en los últimos seis meses en las unidades con hemodiálisis, por ejemplo, el número de pacientes al 1 de enero de 2008 y al 30 de junio de 2008. La cuarta cédula estimó la calidad de la atención mediante la revisión de los expedientes clínicos de los pacientes atendidos en las unidades de hemodiálisis, así como el registro de los tratamientos y exámenes de laboratorio practicados durante los últimos seis meses.

La información fue capturada y validada a través de medidas de frecuencias simples, con el programa estadístico Stata v.10. Para el análisis de las cédulas 1 y 2 se construyeron dos índices a partir de las preguntas establecidas. La medida de resumen fue el promedio por unidad y por indicador. Éstos se ordenaron de mayor a menor (la proporción máxima fue de 100\% y la mínima de $24 \%$ ), y se estratificaron en cinco categorías con los siguientes puntos de corte: $100-90 \%=$ muy buena; $89-80 \%=$ buena; $79-70 \%=$ regular; $69-60 \%=$ mala $\mathrm{y}>60 \%=$ muy mala.

La proporción de unidades incluidas en cada categoría de acuerdo con la cédula 1 fue la siguiente: $21.5 \%, 12.7 \%, 17.7 \%, 13.9 \%$ y $34.2 \%$, respectivamente; y para la cédula $2: 3.6 \%, 10.8 \%, 9.6 \%, 13.3 \%$ y $62.7 \%$, respectivamente. Posteriormente se promediaron ambas proporciones para cada una de las unidades y de nuevo se ordenaron de mayor a menor para agruparse en cada una de las categorías de acuerdo con el puntaje obtenido y con los puntos de corte establecidos. Cabe mencionar que este promedio sólo se pudo realizar en 79 unidades de hemodiálisis, porque en cuatro unidades no se obtuvo la información correspondiente a procesos y resultados (Cédula 1). Finalmente, los resultados de cada cédula se graficaron de manera vectorial por unidad y por indicador y se agruparon en las cinco categorías antes mencionadas.

\section{Resultados}

Se evaluaron 83 unidades médicas con servicio de hemodiálisis, es decir, $27 \%$ del total de unidades identificadas (312). La distribución de las unidades visitadas por entidad federativa se muestra en la figura 1; la mayor proporción de unidades de hemodiálisis se ubicó en el Distrito Federal (16.8\%), seguido de Jalisco $(8.4 \%)$ y Tamaulipas $(6 \%)$. El 37\% de ellas correspondieron al sector privado, 


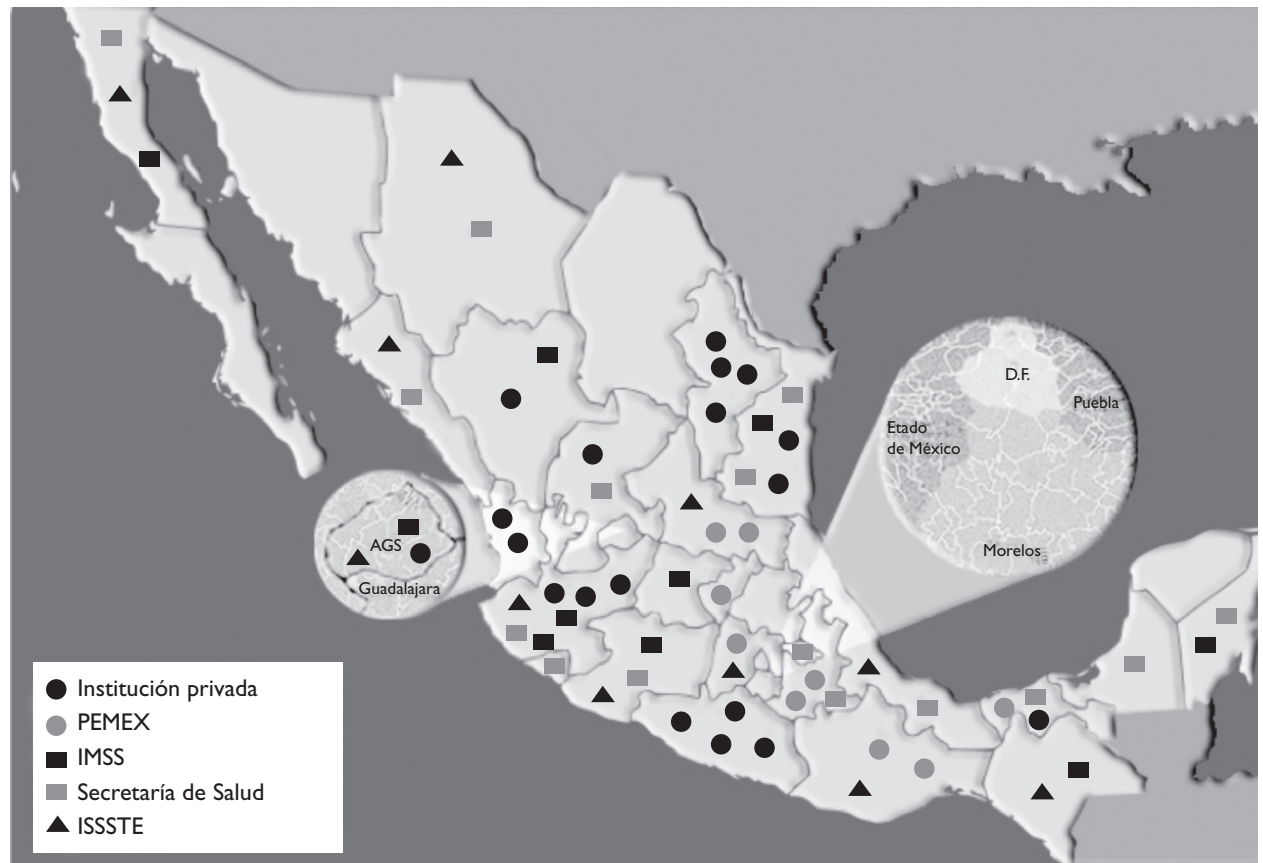

Figura I. Distribución geográfica de las unidades Con hemodiálisis. México, 2008

$22 \%$ a la Secretaría de Salud, 20\% al ISSSTE, $16 \%$ al IMSS, $4 \%$ a PEMEX y $1 \%$ a un hospital universitario.

La evaluación en relación con los procesos y resultados obtuvo un promedio general de $81.9 \%$, con una variabilidad intraunidades entre 0 y $100 \%$ El $34.2 \%$ de las unidades de hemodiálisis obtuvieron un puntaje de 80 o más, lo que dio como resultado que se ubicaran en las categorías buena y muy buena. El $48 \%$ obtuvo un puntaje de 60 o menos, ubicándose en las categorías mala y muy mala. La figura 2 muestra que 19 unidades obtuvieron un promedio de $96.8 \%$, es decir, la evaluación para cada uno de los 19 indicadores fue cercana a $100 \%$, a diferencia de las 27 unidades de la categoría muy mala, donde ninguno de los indicadores alcanzó $100 \%$.

El promedio general en la evaluación de los 19 indicadores de estructura fue de $71.7 \%$, con un valor mínimo de $22.6 \%$ y un valor máximo de $99.1 \%$. La distribución por categoría de acuerdo con los indicadores de estructura fue muy buena $3.6 \%$, buena $10.8 \%$, regular $9.6 \%$, mala $13.3 \%$ y muy mala $62.7 \%$.

En la figura 3 se observa que en la categoría muy buena el número de unidades desciende con respecto al observado en la evaluación de procesos y resultados (2 vs. 19) y el número de unidades clasificadas como muy malas se duplicó (53 vs. 27). El promedio obtenido para la evaluación de los 23 indicadores de estructura en las unidades con categoría muy buena fue de $94.7 \%$ y en la unidades con categoría muy mala de $43 \%$.

A través del promedio de ambas cédulas se concluyó que 19\% de las unidades con servicio de hemodiálisis se ubicaron en las categorías buena y muy buena y $65 \%$ en las categorías mala y muy mala.

En las categorías muy buena y buena se observó que $73 \%$ (11) de las unidades correspondieron al sector privado y únicamente $27 \%$ (4) correspondieron al sector público. La media en las unidades privadas fue de 88.2 y en la unidades públicas fue de 85.5; la diferencia observada no resultó estadísticamente significativa ( $t=$ $0.87, \mathrm{p}=0.4)$.

La evaluación de los registros de información secundaria mostró que $58 \%$ de los pacientes son atendidos en unidades de hemodiálisis clasificadas con puntuación menor a $70 \%$. La proporción fue similar en los pacientes de primera vez (51\%).

La tasa de letalidad fue inversamente proporcional al nivel de las unidades de hemodiálisis, es decir, en las unidades clasificadas como muy malas la tasa de letalidad fue casi cuatro veces más alta que en las unidades consideradas como muy buenas ( 21.0 vs. $5.2 \%$ ) (cuadro I). La pérdida de pacientes en las unidades de hemodiálisis varió entre 25 y 50\%, lo cual hace suponer que la tasa de letalidad está subestimada. 

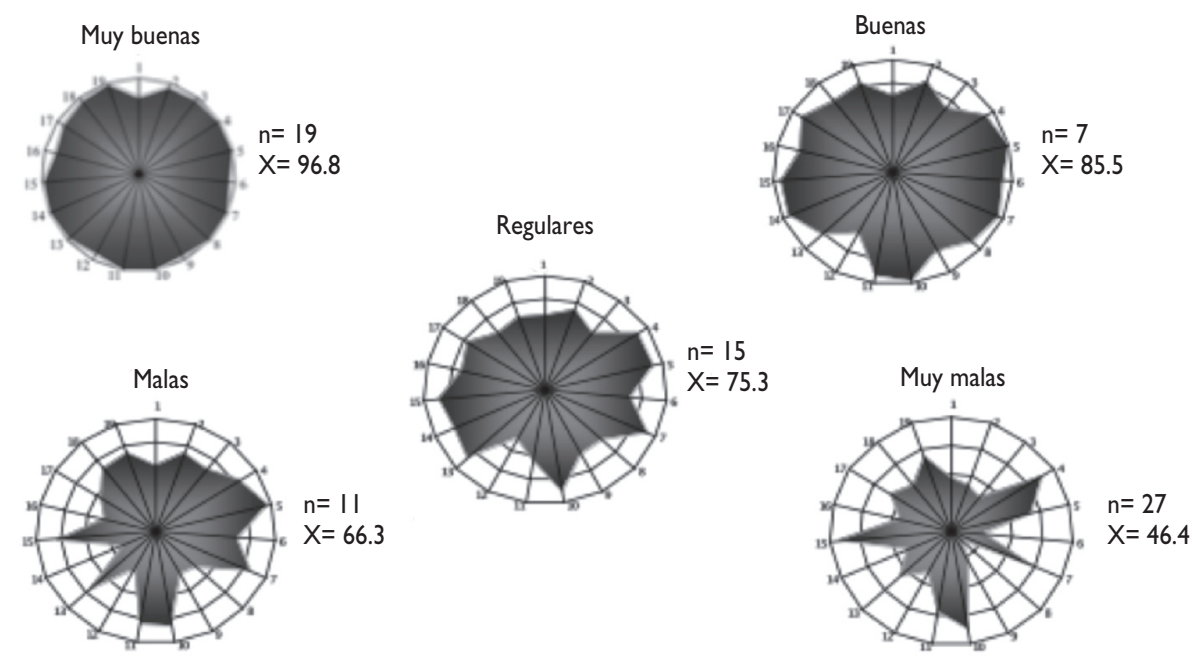

I. Código de ética

2. Carta de los derechos de los pacientes

3. Quejas y sugerencias

4. Residuos biológicos infecciosos peligrosos

5. Atención del paciente

6. Planeación anual

7. Cumplimiento de lineamientos

8. Referencia y contrarreferencia de pacientes

9. Urgencias

10. Tratamiento seguro, oportuno y con capacidad resolutiva

II. Expediente clínico

12. Carta de los derechos de los pacientes

13. Servicio de apoyo diagnóstico

14. Personal médico y paramédico

15. Seguridad y eficiencia del equipo

16. Programa de mejora continua de calidad

17. Medición y obtención de indicadores de resultados de sistemas de información

18. Resultados

19. Instalaciones

Figura 2. Evaluación de procesos y resultados en las unidades de hemodiálisis, según Categoría. MéXico, 2008.

Finalmente, se revisó el expediente clínico de los pacientes a fin de analizar los exámenes de laboratorio realizados (hemoglobina, ferritina, fósforo sérico, albúmina y hormona paratifoidea), el tipo de tratamiento recibido para corregir la anemia (eritropoyetina o darbopoyetina) y la determinación del índice $\mathrm{Kt} / \mathrm{V}$, registrados en los últimos seis meses.

En total se revisaron los expedientes de 818 pacientes en 83 unidades con hemodiálisis; de ellos, $18 \%$ correspondieron a unidades con categoría buena y muy buena y $67 \%$ a unidades con categoría mala y muy mala. El número total de sesiones evaluadas fue de 25451 . El promedio de sesiones por paciente en los seis meses evaluados fue de 1.2 por semana con un intervalo entre 0.8 y 1.3 al interior de las cinco categorías de las unidades de hemodiálisis.

En relación con los exámenes de laboratorio, se observó que en $57 \%$ de las sesiones no se registraron los parámetros bajo estudio durante todo el período de seguimiento. De las sesiones que sí tenían algún registro de exámenes de laboratorio, solamente 6.5\% correspondió a hemoglobina. Las proporciones en el resto de los exámenes fueron aún menores, y única- mente $0.1 \%$ (25) del total de las sesiones revisadas registraron resultados de los cinco exámenes de laboratorio evaluados.

En los pacientes con tres sesiones, el reporte de los exámenes de laboratorio fue muy similar, siendo la hemoglobina la que se reportó con mayor frecuencia $(4.7 \%)$, y por otra parte, ninguna de las sesiones en este grupo de pacientes reportó la totalidad de los exámenes evaluados (cuadro II).

Para determinar la efectividad de la hemodiálisis se requiere, además de los exámenes de laboratorio ya citados, el índice Kt/V. Éste permite medir el aclaramiento total de urea $\left(K^{*} t\right)$, donde $K$ representa el aclaramiento de urea del dializador, t el tiempo de duración de la hemodiálisis, dividido por el volumen de distribución de la urea, el cual equivale aproximadamente al agua corporal total. El valor óptimo del $\mathrm{Kt} / \mathrm{V}$, según las Guías Europeas de Buenas Prácticas, debe ser igual o superior a 1.2 para $80 \%$ de los pacientes tratados. En este estudio se observó que únicamente $54.9 \%$ de expedientes tenían al menos un registro de $\mathrm{Kt} / \mathrm{V}$ durante los seis meses considerados para el análisis, y de ellos $46.5 \%$ reportaron valores inferio- 

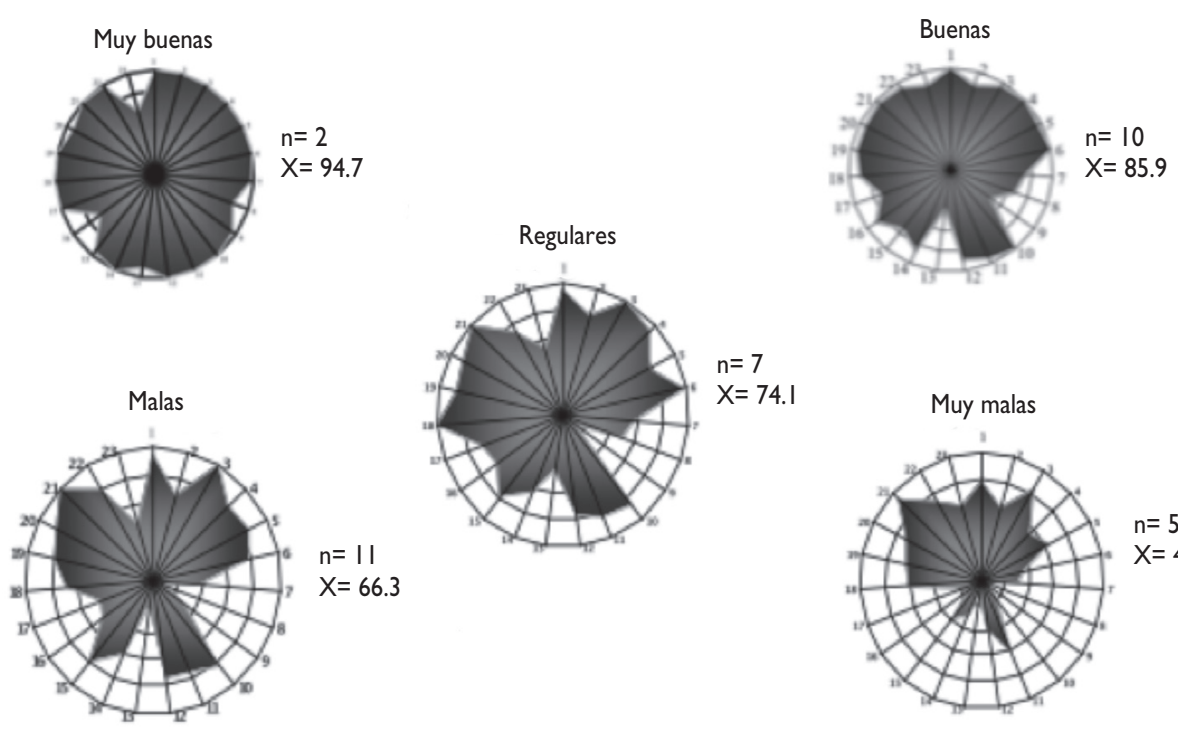

I. Accesibilidad a la unidad de hemodiálisis

14. Cuarto séptico

15. Consultorios médicos

2. Vías de acceso del transporte público

3. Acceso en la unidad de hemodiálisis

4. Área de recepción-admisión-información

17. Vestidores del personal de salud

5. Sala de espera

6. Área secretarial

7. Servicios sanitarios para pacientes

8. Servicios sanitarios para pacientes discapacitados

18. Baños del personal de salud

19. Instalaciones físicas, eléctricas, de agua, extinguidores de fuego y calefacción

9. Vestidores para pacientes

20. Verificación de las instalaciones físicas, eléctricas, de agua, extinguidores de fuego y calefacción

10. Zona de camillas y sillas de ruedas

II. Sala de hemodiálisis

12. Sala de mantenimiento

I. Acceso o disponibilidad de insumos inherentes

22. Realización de exámenes de laboratorio

13. Ropería

23. Realización de exámenes de gabinete

Figura 3. Evaluación de la estructura en las unidades de hemodiálisis, según categoría. México, 2008

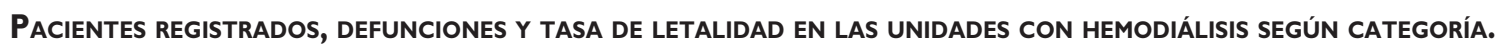 MÉXICO, ENERO-JUNIO de 2008}

\begin{tabular}{|c|c|c|c|c|c|c|c|c|}
\hline Categoría & $\begin{array}{c}\text { Pacientes Registrados } \\
\qquad \text {-0I-2008 }\end{array}$ & $\%$ & Pacientes $I^{a}$ vez & $\%$ & $\begin{array}{c}\text { Pacientes registrados } \\
30-06-2008\end{array}$ & \% de pérdida & Defunciones & $\begin{array}{c}\text { Tasa Letalidad } \\
\times 100\end{array}$ \\
\hline Muy buena & 1090 & 17.4 & 740 & 16.2 & 1314 & 25 & 68 & 5.2 \\
\hline Buena & 461 & 7.4 & 626 & 13.7 & 534 & 49 & 42 & 7.9 \\
\hline Regular & 1063 & 17.0 & 857 & 18.8 & 1169 & 37 & 74 & 6.3 \\
\hline Mala & 1765 & 28.2 & 884 & 19.4 & 2447 & 0 & 245 & 10.0 \\
\hline Muy mala & | 882 & 30.0 & $\mathrm{I}, 453$ & 31.9 & 1516 & 50 & 318 & 21.0 \\
\hline Total & 6261 & 100 & & 100 & 6980 & 31 & 747 & 10.7 \\
\hline
\end{tabular}

Fuente: Registros de las unidades de hemodiálisis

res al valor recomendado por las guías terapéuticas españolas $(K t / V \geq 1.2)$.

Por último, la evaluación de los recursos humanos de las unidades de hemodiálisis mostraron alta variabilidad al interior de las categorías. En aquellas denominadas buenas y muy buenas se observó que en promedio disponían hasta de cuatro médicos nefrólogos, dos médicos generales y quince enfermeras. En 
Cuadro II

Distribución PROPORCIONAL de LOS EXÁMENES de LABORATORIO Y KT/V EN LAS SESIONES de heMOdiÁLISIS DE LOS PACIENTES INCLUIDOS EN EL ESTUdio. MÉXICO, 2008

\begin{tabular}{|c|c|c|c|c|c|c|c|c|}
\hline & $\begin{array}{l}\text { Sin ningún } \\
\text { estudio }\end{array}$ & $K t / V$ & Hemoglobina & Ferritina & Albúmina & $\begin{array}{l}\text { Hormona } \\
\text { paratiroidea }\end{array}$ & $\begin{array}{l}\text { Fósforo } \\
\text { sérico }\end{array}$ & $\begin{array}{c}\text { Num. de sesiones con determinación } \\
\text { de todos los estudios* }\end{array}$ \\
\hline Total de sesiones $(n=2545 \mathrm{I})$ & 14478 & 10161 & 1663 & 303 & 1021 & 88 & 991 & 25 \\
\hline$\%$ & 56.9 & 39.9 & 6.5 & 1.2 & 4.0 & 0.3 & 3.9 & 0.1 \\
\hline 16 pacientes con tres sesiones & 305 & 340 & 33 & 2 & 6 & 0 & 16 & 0 \\
\hline$\%$ & 43.4 & 48.2 & 4.7 & 0.3 & 0.9 & 0 & 2.3 & 0 \\
\hline
\end{tabular}

cambio, las unidades con categorías mala y muy mala contaban en promedio con menos de un nefrólogo, con un médico general por cada dos unidades y con siete enfermeras.

\section{Discusión}

Hasta donde sabemos, este es el primer estudio que permite apreciar el estado funcional y estructural de las unidades de hemodiálisis en México; los resultados permiten apreciar sus fortalezas y debilidades. En primer lugar, se carece de un directorio con el registro de las unidades de hemodiálisis a nivel nacional, mismo que se fue conformando durante la investigación; al momento actual se han identificado más de 300 unidades en nuestro país. Sin embargo, es muy probable que exista un número mayor sobre todo de pequeñas dimensiones y de carácter privado que son más difíciles de identificar.

En segundo lugar se detectó la falta de información sobre el funcionamiento y la estructura de las unidades de hemodiálisis, así como ausencia de registros que permitan evaluar su efectividad a través de la evolución y sobrevida de los pacientes sometidos a dicha terapia de sustitución. Dentro de este rubro, el estudio también mostró un panorama sumamente preocupante pues al menos la mitad de las unidades no cumplen con los criterios de proceso y resultado para la evaluación de establecimientos de atención médica de hemodiálisis establecidos por el Consejo de Salubridad, ${ }^{1}$ sobre todo las unidades públicas. Esta situación se agrava aún más al evaluar la infraestructura de las unidades, donde tres cuartas partes de ellas obtuvieron puntajes menores a $70 \%$.

Otro aspecto que llamó la atención fue la falta de procesos para la referencia y contarreferencia de los pacientes, hecho que explica de manera indirecta el retraso en el ingreso a estas unidades y por ende, la falta de respuesta al tratamiento.

La mayoría de los expedientes clínicos no contaron con información mínima solicitada, y los que sí la registraban presentaban fallas de calidad en el manejo terapéutico del paciente, como el hecho de que $49 \%$ de los expedientes revisados registraron menos de una sesión de hemodiálisis a la semana, y únicamente 2\% (16 pacientes) cumplió con la norma establecida de tres sesiones por semana. Asimismo, se observó la falta de exámenes de laboratorio para evaluar el estado de los pacientes previo a la sesión de hemodiálisis aún en los casos en que asistían a tres sesiones, donde menos de $5 \%$ de las mismas contaron con determinación de hemoglobina y $0.3 \%$ con determinación de ferritina, a pesar de que ambos exámenes deben realizarse con frecuencia mensual y bimestral, respectivamente, de acuerdo con la normatividad.

La determinación de $\mathrm{Kt} / \mathrm{V}$ es otro parámetro de gran importancia en la determinación de la dosis de diálisis y su determinación oportuna se refleja en la morbilidad y la mortalidad. Con base en la guía práctica para hemodiálisis: Dialisis Outcomes Quality Iniciative (DOQI) de la National Kidney Fundation, ${ }^{8-11}$ se recomienda un $\mathrm{Kt} / \mathrm{V}$ igual o mayor a 1.2, y evaluarlo al menos mensualmente.

El estudio también reflejó la falta de calidad en la atención médica durante el proceso de la hemodiálisis. Más de la mitad de las sesiones evaluadas no contaron con la determinación de Kt/V mínima mensual, y en aquellas que síla tuvieron, menos de la mitad reportaron valores por debajo de los recomendados en las guías de manejo terapéutico para hemodiálisis en régimen de tres sesiones semanales (Kt/V igual o superior a 1.2). ${ }^{11-12}$ Por todas estas razones, resultó imposible valorar la sobrevida 
y los parámetros de calidad de atención tanto de manera individual como por unidad.

En relación con el acceso vascular (fístula arteriovenosa), se puede comentar que la proporción de los pacientes identificados con acceso vascular en las unidades de hemodiálisis resultó muy inferior al recomendado por el estudio Dialysis Outcomes and Practice Pattern Study (DOPPS) ${ }^{13}$ en Europa, donde 66\% de los pacientes inician la hemodiálisis con un acceso vascular autólogo. Las guías K/DOQI ${ }^{7}$ recomiendan que $50 \%$ de los pacientes que inician hemodiálisis deben tener un acceso vascular. En este estudio sólo 8\% de los pacientes tuvieron este acceso vascular.

La falta de personal calificado (nefrólogos) durante el proceso de la hemodiálisis fue muy evidente en las unidades, donde los 10821 pacientes registrados (al 30 de junio de 2008) fueron atendidos por 180 médicos nefrólogos, lo que dio en promedio 57 pacientes por nefrólogo, cifra que aumentó a 73 pacientes por nefrólogo en las unidades clasificadas dentro de la categoría muy mala. Esta situación contrasta con la norma que recomienda que cada centro de diálisis debería contar con al menos un nefrólogo por cada 40-50 pacientes en tratamiento. ${ }^{4}$

En el caso del personal de enfermería, de acuerdo con los indicadores de algunos países de la unión europea, ${ }_{14}^{14}$ se requiere una enfermera por cada 4 o 5 máquinas de hemodiálisis y un auxiliar de enfermería por cada 8 a 10 puestos de hemodiálisis; en este estudio se observó que en promedio existe una enfermera por máquina de hemodiálisis, aun en las unidades con categorías mala y muy mala; en cambio, la relación auxiliares de enfermería por máquina de hemodiálisis coincidió con la norma, con nueve auxiliares por máquina.

Como se pudo observar, en nuestro país la mayoría de las unidades de hemodiálisis no cuentan con las características, proceso e infraestructura mínima recomendada por las normas internacionales y nacionales. Finalmente, se deben tomar en consideración los resultados obtenidos y el costo elevado de la hemodiálisis, lo cual representa un dispendio de recursos para el sistema de salud, ya que el beneficio es muy pobre en la mayoría de las unidades debido a deficiencias de calidad en las unidades: $70 \%$ de ellas reportaron tasas de letalidad muy elevadas.

Con base en los resultados de este trabajo se hacen las siguientes recomendaciones:

1. Capacitar a los responsables de las unidades de hemodiálisis para la planeación anual de manera oportuna a fin de contar con los recursos necesarios para proporcionar la atención médica.
2. Organizar los servicios de salud para evitar la saturación de los mismos y mejorar la oferta de hemodiálisis.

3. Conformar un registro nacional confiable con los pacientes tratados en las unidades de hemodiálisis que permita identificar no sólo la prevalencia de este padecimiento sino también las características de los pacientes, la respuesta al tratamiento y la sobrevida de los mismos.

4. Revisar o establecer los procesos para la referencia y contrarreferencia a fin de que los pacientes reciban el tratamiento oportuno y óptimo.

5. Mejorar la equidad en el sistema de salud para que la población con menos recursos tenga acceso a la hemodiálisis; se estima que actualmente más de la mitad de los pacientes no reciben dicho tratamiento. ${ }^{3}$

6. Mejorar e incrementar la capacidad en la infraestructura de las unidades de hemodiálisis para incrementar la calidad de la misma.

7. Establecer programas de trabajo entre el sector salud y las universidades a fin de incrementar la formación de médicos especialistas en nefrología, ya que al momento resultan insuficientes. Asimismo, es importante implementar estrategias para la distribución equitativa de dicho personal y evitar la concentración en las grandes ciudades.

8. Revisar la legislación que existe en relación con la hemodiálisis (Norma Oficial Mexicana NOM-171SSA1-1998 para la práctica de hemodiálisis) a fin de hacerla acorde con el panorama epidemiológico que prevalece en nuestro país.

Con los datos y recomendaciones presentados, las instituciones públicas en cada entidad federativa y también en el nivel federal, podrán sustentar mejor sus acciones programáticas y ajustar las políticas de identificación y control de la ERC en México y con ello mejorar la calidad en su atención.

Declaración de conflicto de intereses: Los autores declararon no tener conflicto de intereses.

\section{Referencias}

I. Atkins RC. The epidemiology of chronic kidney disease. Kidney Int 2005: SI4-SI8.

2. Paniagua R, Ramos A, Fabian R, Lagunas J, Amato D. Chronic kidney disease and dialysis in Mexico. Perit Dial Int 2007;27:405-409.

3. Paniagua R, Amato D, Vonesh E, Correa-Rotter R, Ramos A, Moran $J$, et al. Effects of increased peritoneal clearances on mortality rates in peritoneal dialysis: ADEMEX, a prospective, randomized, controlled trial. J Am Soc Nephrol 2002; I3:1307-I320. 
4. Consejo de Salubridad General. Comisión para la certificación de establecimientos de Servicios de Salud. Estándares de procesos y resultados para la evaluación de establecimientos de atención médica de hemodiálisis. FR-EM-03-05. [Consultado 2008 abril 23] Disponible en: http://www.csg.salud.gob.mx/descargas/pdfs/certificacion/establecimientos/ Hemodialisis/HEMOD_PROCESOSYRESULT.pdf

5. Alcalde G, Martín de Francisco AL, Fernández A, Conde JL. Dotación de personal para centros de hemodiálisis ambulatoria. Nefrologia 2006;26: SII-SI4.

6. Angoso M, Alcalde G, Alvarez-Ude F, Arenas MD. Gestión de calidad en hemodiálisis. Nefrologia 2006;26 supl 8:S73-S87.

7. Solozábal C, Pérez-García R, Martí A. Características estructurales de las unidades de hemodiálisis. Nefrología 2006;26 supl 8:S5-SI0.

8. NKF-DOQI clinical practice guidelines for hemodialysis adequacy.

Am J Kid Dls 1997; 30: S22-S57.

9. K/DOQI clinical practice guidelines on hypertensión and antihypertensive agents in chronic kidney disease. Am J Kidney Dis 2004; 43: SI-S290.
10. Nacional Kidney Foundation K/DOQI clinical practice guidelines on managing dyslipemias in chronic kidney disease. Am J Kidney Dis 2003; 4I: SI-S77.

II. National Kidney Foundation K/DOQI clinical practice guidelines for bone metabolism and disease in Chronic Kidney Disease. Am J Kidney Dis 2003; 42: SI-S2OI.

12. Maduell F, García M, Alcázar R. Dosificación y adecuación del tratamiento dialítico. Nefrologia 2006;26: SI5-S2I.

13. Piera L, Cruz JM, Braga-Gresham JL, Eichleay MA, Pisoni RL, Port FK. Estimación, según el estudio DOPPS, de los años de vida de pacientes atribuibles a las prácticas de hemodiálisis modificables en España. Nefrología 2007; 27: 496-504.

14. Cruz JM, Piera L, Bragg-Gresham JL, Feldman H, Port FK. Resultados del Estudio Internacional de Hemodiálisis DOPPS en Europa y España. Nefrología 2003; 23: 437-443. 